\title{
Utility of ferritin and inflammatory biomarkers in the diagnosis of different stages of breast cancer
}

\author{
Anila George, MSc, Zachariah Bobby, PhD, Biswajit Dubashi, DM.
}

\begin{abstract}
الأهداف : تقييم فائدة الفيريتين والمؤشرات الحيوية للالتهاب في تشخيص مراحل

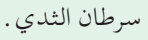

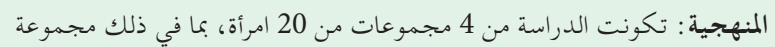

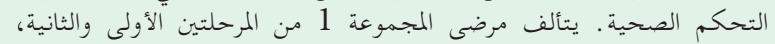

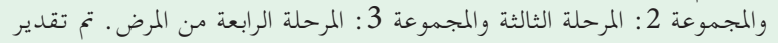

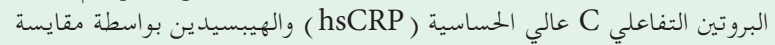

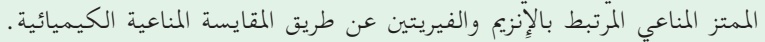

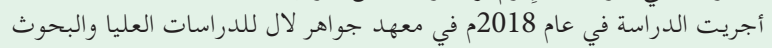

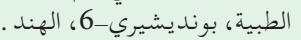

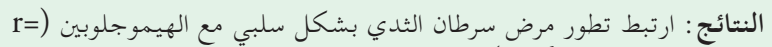

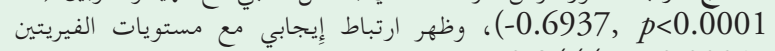

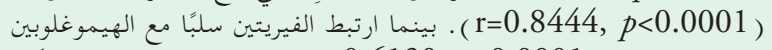

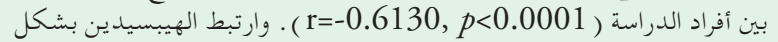

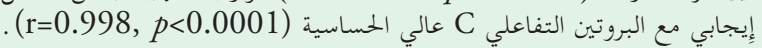

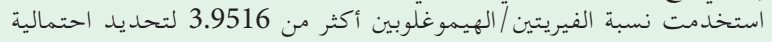

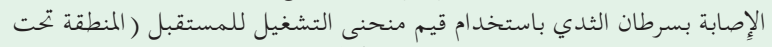

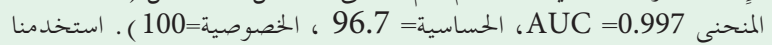

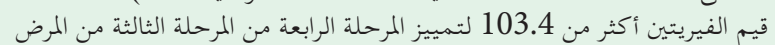

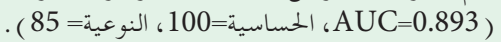

الخلاصة: يعد كلاً من الفيريتين والفيريتين /الهيموغلوبين مفيدان في معرفة تشخيص مراحل سرطان الثدي.
\end{abstract}

Objectives: To assess the utility of ferritin and inflammatory biomarkers in the diagnosis of stages of breast cancer.

Methods: The study consisted of 4 groups of 20 women, including the healthy control. The patients of group 1 comprised of stages I and II, group 2: stage III and group 3: stage IV of the disease. High sensitive C-reactive protein (hsCRP) and hepcidin were estimated by enzyme linked immunosorbent assay and ferritin by chemiluminescence immunoassay. The study was carried out in 2018 at Jawaharlal Institute of Postgraduate Medical Education and Research, Puducherry-6, India.

Results: The breast cancer disease progression correlated negatively with hemoglobin $(\mathrm{Hb}) \quad(\mathrm{r}=-0.6937$, $p<0.0001)$ and with ferritin levels, had a positive correlation $(\mathrm{r}=0.8444, p<0.0001)$. Ferritin negatively correlated with $\mathrm{Hb}$ among the subjects of the study $(\mathrm{r}=-0.6130, p<0.0001)$. Hepcidin correlated positively with hsCRP $(\mathrm{r}=0.998, p<0.0001)$. The ferritin/Hb ratio $>3.9516$ was used to identify the possibility of breast cancer utilizing the receiver operator curve values (area under curve $[\mathrm{AUC}]=0.997$, sensitivity: 96.7, specificity:100). Ferritin values $>103.4$ were used to differentiate stage 4 from stage 3 of the disease (AUC: 0.893 , sensitivity: 100, specificity: 85 ).

Conclusion: Ferritin and ferritin/ $\mathrm{Hb}$ are helpful in the differential diagnosis of stages of breast cancer.

Keywords: breast cancer, inflammation, ferritin, hepcidin, differential diagnosis, disease progression

Saudi Med J 2021; Vol. 42 (8): 825-831

doi: $10.15537 /$ smj.2021.42.8.20210244

From the Department of Biochemistry (George, Bobby), and from the Department of Medical Oncology (Dubashi), Jawaharlal Institute of Postgraduate Medical Education and Research, Puducherry, India.

Received 8th April 2021. Accepted 27th June 2021.

Address correspondence and reprint request to: Dr Zachariah Bobby, Department of Biochemistry, Jawaharlal Institute of Postgraduate Medical Education and Research, Puducherry, India.E-mail: zacbobby@yahoo.com ORCID ID: http://orcid.org/0000-0002-9438-7319

$\mathrm{I}^{\mathrm{n}}$ the pathogenesis of breast cancer, inflammation has an important impact. Proinflammatory state leads to the generation of a tumor-related inflammatory microenvironment. Identification of factors for the diagnosis of various breast cancer stages can help the management of the disease. Studies have reported the usefulness of inflammatory biomarkers for predicting the prognosis and outcome of breast cancer. ${ }^{1}$ Only limited 
studies have addressed their importance in diagnosing advanced states of breast cancer. This is of great concern since metastasis to various organs can affect both the overall survival and disease-free survival of patients. In this study, we assessed the utility of inflammatory markers, ferritin, high sensitive C-reactive protein (hsCRP) and hepcidin in the diagnosis of different stages of breast cancer. Liver produces the acute phase reactant, $\mathrm{C}$ reactive protein (CRP). The transcriptional regulation of CRP synthesis is primarily regulated by the Interleukin-6 synthesized and secreted from the tumor. ${ }^{2}$ The microenvironment of tumor cells can also initiate inflammation. This enables tumor progression via invasion and metastasis. ${ }^{3,4}$ Therefore, increasing CRP levels with advanced stages of breast cancer at the time of diagnosis is expected. In the pathogenesis of breast cancer, the deranged iron homeostasis plays a major role. ${ }^{5}$ The storage form of iron inside the cells is ferritin and is also found in the circulation. The tissue and systemic levels of ferritin increase in breast cancer and are associated with disease progression. ${ }^{6}$ The macrophages that infiltrate breast tumors can release ferritin, which is implicated in tumorigenesis by triggering inflammation.?

The serum ferritin is elevated in inflammation and cancers. This is related to its enhanced synthesis in macrophages. During inflammation, hepcidin promotes the entry of iron into the macrophages. ${ }^{6}$ Therefore, in breast cancer, there are strong associations among iron homeostasis, anemia, inflammation and hepcidin release. Biochemical derangements related to low-grade inflammation is expected to increase in the disease progression in breast cancer. Assessment of the biochemical parameters like ferritin, hepcidin, hsCRP, and ferritin/Hb ratio would be helpful in the differential diagnosis of different stages of breast cancer.

The present study was carried out with the hypothesis that elevated serum inflammatory biomarkers would serve as additional parameters in breast cancer diagnosis and staging.

Methods. Consecutive breast cancer patients attending the Oncology Clinic in the Department of Medical Oncology, Jawaharlal Institute of Postgraduate Medical Education and Research (JIPMER), Puducherry,

Disclosure. Authors have no conflict of interests, and the work was not supported or funded by any drug company.
India were recruited for the study. The recruitment was carried out based on the set inclusion and exclusion guidelines. As controls, we recruited apparently healthy women. Ethical clearance to conduct this work was obtained from the Institute Ethics Committee (IEC), JIPMER, and the participants have signed the informed consent. This was a cross-sectional analytical study. The study was conducted in the year 2018 at JIPMER, Puducherry, India. The study consisted of 4 groups of 20 women, each in 30-60 years of age, including the healthy control. The sample size was calculated using the reported differences in the mean hsCRP levels between controls and 3 groups of breast cancer patients. ${ }^{8}$ Assuming alpha error of 5\%, power of $90 \%$ and standard deviation within each group as 2 , we required a minimum of 15 individuals in each group. Based on the American Joint Committee on Cancer guidelines (AJCC), the staging of breast cancer was carried out. Based on stages, the newly diagnosed treatment-naive breast cancer patients were segregated into 3 groups. The patients of group 1 comprised of stages I and II of the disease. Group 2 consisted of patients in stage III, and the stage IV patients were included in group 3. Subjects with a history of any chronic or acute inflammatory diseases, on anti-inflammatory medication, diabetes mellitus, hypertension and liver or kidney diseases were excluded from the study. We did not consider ER, PR and human epidermal growth factor receptor 2 (HER2) neu expressions as inclusion and exclusion criteria for the recruitment.

Collection of blood samples. Fasting blood $(5 \mathrm{ml})$ was drawn from all subjects of the study. The samples were collected in EDTA tubes for hemogram. For the assay of biochemical parameters, serum was used, which was stored at $-40^{\circ} \mathrm{C}$ until the analysis.

Biochemical assays. Serum hsCRP was estimated by ELISA using commercially available kits (DBC, Canada). Serum hepcidin was estimated using ELISA kit from Wuhan Fine Biotech co. Ltd, China. Estimation of serum ferritin was carried out by chemiluminescence immunoassay system in Seimens Advia Centaur CP.

Statistical analyses. GraphPad Prism version 8.0.2 was used for the statistical analyses. KolmogorovSmirnov test was used for checking the distribution of data. The data which did not follow a normal distribution are presented as median (interquartile range) and Kruskal-Wallis test was used for comparison between the groups. The data which followed normal distribution are presented as mean $\pm S D$ and were compared with ANOVA. MedCalc software was used for plotting the ROC curves. The results were considered significant when the p-value was found to be $<0.05$. 
Results. The mean age in years in different groups were 49 in control, stages I, 2 and 4 and 46 in stage 3. Twelve participants were post-menopausal in each group except in the stage 3 group, where 9 patients were post-menopausal. The number of triple-negative breast cancer patients were 4, 3 and 2 respectively in groups 1,2 and 3 .

Comparison of study parameters between controls and all breast cancer patients. The $\mathrm{Hb}$ was found to be lower, and the levels of ferritin, hepcidin and hsCRP were found higher among breast cancer patients of our study (Table1).

Comparison of study parameters between controls and breast cancer patients in different stages of the disease. There was a significant increase in the levels of serum ferritin across the different groups of study. A trend for decrease in $\mathrm{Hb}$ and increase in hepcidin and hsCRP with increasing stages of the disease was observed among the breast cancer patients. The results are presented in Table 2. There was a significant increase in the neutrophil/lymphocyte ratio among the stage 4 breast cancer patients when compared to all the other groups.

The comparison of $\log$ ferritin/ $\mathrm{Hb}$ ratio between the study groups is further represented in Figure 1.

Comparison of lipid profile between controls and breast cancer patients in different stages of the disease. The lipid profile did not differ much from the normal

Table 1 - Comparison of study parameters between controls and breast cancer patients.

\begin{tabular}{lccc}
\hline Parameters & Control $(\mathbf{n}=20)$ & Cases $(\mathbf{n}=60)$ & $P$-value \\
\hline $\mathrm{Hb}(\mathrm{g} / \mathrm{dL})$ & $12.40(12.10-12.50)$ & $10.95(10.33-11.80)$ & $<0.0001^{*}$ \\
$\mathrm{HsCRP}(\mathrm{ng} / \mathrm{ml})$ & $3686(463.2-7628)$ & $6008(2653-19032)$ & $0.0326^{*}$ \\
$\mathrm{Hepcidin}(\mathrm{pg} / \mathrm{ml})$ & $73710(9263-512749)$ & $120165(53060-640558)$ & $0.0397^{*}$ \\
Ferritin $(\mathrm{ng} / \mathrm{ml})$ & $29.70(25.73-45.70)$ & $82.95(52.65-167.9)$ & $<0.0001^{*}$ \\
Ferritin/Hb ratio & $2.458(2.035-3.757)$ & $7.590(4.859-16.02)$ & $<0.0001^{*}$ \\
\hline
\end{tabular}

All data expressed as median (Inter Quartile Range). ${ }^{*} P$-value $<0.05$ considered as significant

Table 2 - Comparison of study parameters between controls and breast cancer patients in different stages of the diseases.

\begin{tabular}{|c|c|c|c|c|}
\hline Parameters & Control $(n=20)$ & Stage $1 \& 2(n=20)$ & Stage $3(n=20)$ & Stage $4(n=20)$ \\
\hline $\mathrm{Hb}(\mathrm{g} / \mathrm{dl})$ & $\begin{array}{c}12.40 \\
(12.10-12.50)\end{array}$ & $\begin{array}{c}11.65 \\
(10.68-12.33)^{*}\end{array}$ & $\begin{array}{c}11.20 \\
(10.13-11.78)^{\dagger \dagger \dagger \dagger}\end{array}$ & $\begin{array}{c}10.50 \\
(10.23-10.88)^{\S, \text { 赫 }}\end{array}$ \\
\hline Hepcidin (pg/ml) & $\begin{array}{c}73710 \\
(9263-152564)\end{array}$ & $\begin{array}{c}87725 \\
(44175-179148)\end{array}$ & $\begin{array}{c}122348 \\
(30678-367928)\end{array}$ & $\begin{array}{c}224315 \\
(78415-457778)^{\ddagger}\end{array}$ \\
\hline hsCRP (ng/ml) & $\begin{array}{c}3686 \\
(463.2-7628)\end{array}$ & $\begin{array}{c}4386 \\
(2209-8957)\end{array}$ & $\begin{array}{c}6117 \\
(1534-18396)\end{array}$ & $\begin{array}{c}11216 \\
(3921-22889)^{\ddagger}\end{array}$ \\
\hline Ferritin (ng/ml) & $\begin{array}{c}29.70 \\
(25.73-45.70)\end{array}$ & $\begin{array}{c}56.35 \\
(50.73-78.55)^{* *}\end{array}$ & $\begin{array}{c}66.90 \\
(50.23-96.25)^{\dagger \dagger \dagger}\end{array}$ & 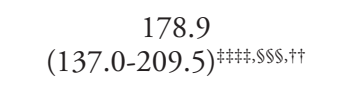 \\
\hline Ferritin/Hb ratio & $\begin{array}{c}2.458 \\
(2.035-3.757)\end{array}$ & $\begin{array}{c}5.501 \\
(4.291-6.713)^{* *}\end{array}$ & $\begin{array}{c}5.580 \\
(4.737-8.759)^{\dagger \dagger \dagger}\end{array}$ & 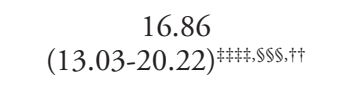 \\
\hline Log ferritin/Hb ratio & $\begin{array}{c}0.1184 \\
(0.1133-0.1343)\end{array}$ & $\begin{array}{c}0.1571 \\
(0.1473-0.1652)^{* *}\end{array}$ & $\begin{array}{c}0.1635 \\
(0.1490-0.1840)^{\dagger \dagger \dagger}\end{array}$ & 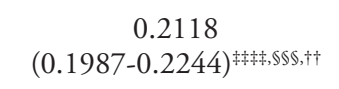 \\
\hline $\mathrm{N} / \mathrm{L}$ ratio & $\begin{array}{c}1.90 \\
(1.467-2.225)\end{array}$ & $\begin{array}{c}1.60 \\
(1.194-2.211)\end{array}$ & $\begin{array}{c}1.71 \\
(1.222-2.298)\end{array}$ & 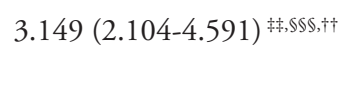 \\
\hline \multicolumn{5}{|c|}{ 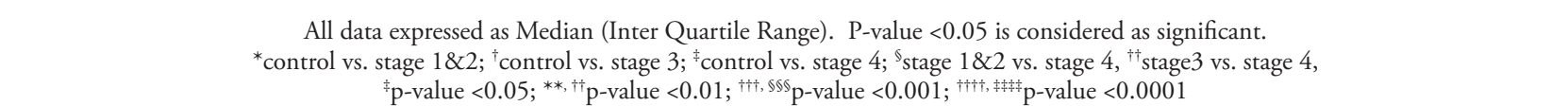 } \\
\hline
\end{tabular}


range among the different stages of the breast cancer except in stage 3. The results are presented in Table 3.

Relationship between study parameters and disease progression. The disease progression significantly correlated negatively with $\mathrm{Hb}$ (Correlation coefficient= -0.6937, $\mathrm{p}<0.0001)$ and positively with serum ferritin levels (Correlation coefficient $=0.8444, p<0.0001$ ). Hemoglobin negatively correlated with ferritin among the subjects of the study (Correlation coefficient= $-0.6130, p<0.0001$, Figure 2). The serum levels of Hepcidin correlated positively with hsCRP which is a marker of inflammation (Correlation coefficient= $0.998, p<0.0001$ ).

Utility of serum ferritin levels in the diagnosis and staging of breast cancer. We evaluated the usefulness of ferritin and ferritin/ $\mathrm{Hb}$ ratio in the diagnosis of different stages of breast cancer by receiver operating characteristic (ROC) analyses. In the absence of any comorbidities, ferritin/Hb ratio $>3.9516$ were used to identify the possibility of breast cancer utilizing the ROC curve values (AUC=0.997, sensitivity: 96.7, specificity:100). Similarly, ferritin values $>103.4$ were used to differentiate stage 4 from stage 3 of the disease (AUC: 0.893, sensitivity: 100, specificity: 85 ). These results are presented in Figure 3.

Discussion. The anatomic extent of the disease was the only consideration for breast cancer staging until recently. However, of late, it has been realized that biological factors are also essential to determine prognosis and to take decisions on treatment. ${ }^{3}$ There are 2 staging systems, according to the American Joint Committee on Cancer's Staging System (8th edition) for breast cancer. The size of the tumor, metastasis and the nodal status are the considerations for anatomic staging. However, in the prognostic staging, which is

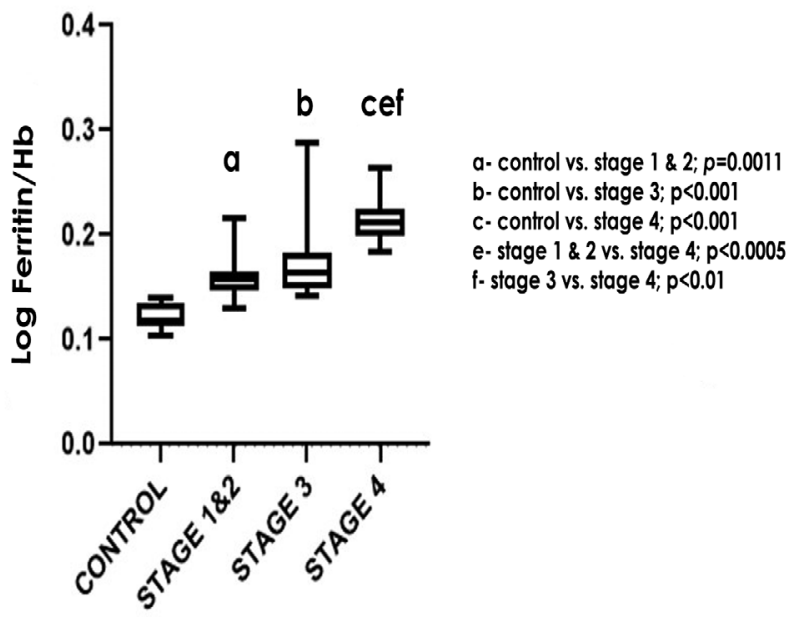

Figure 1 - Comparison of $\log$ ferritin/ hemoglobin $(\mathrm{Hb})$ ratio between the study groups

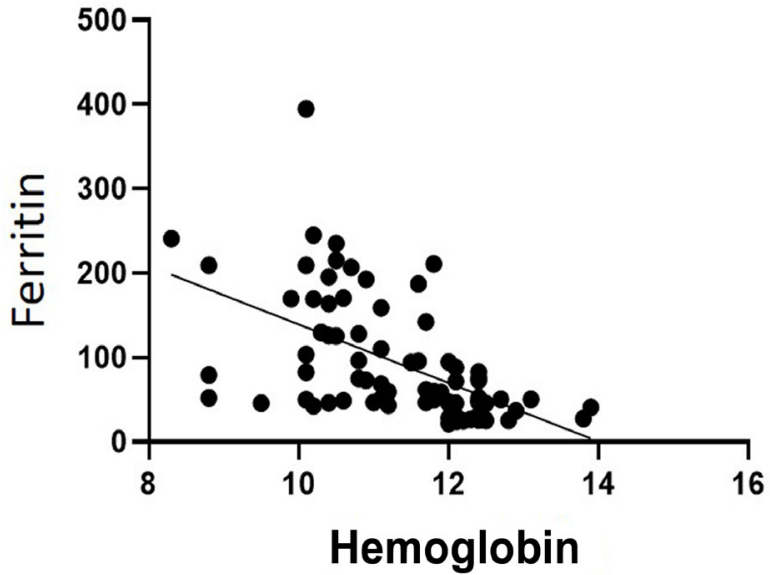

Figure 2 - Correlation of hemoglobin with serum ferritin levels (correlation coefficient $=-0.6130, \mathrm{p}<0.0001)$

Table 3 - Comparison of lipid profile between controls and breast cancer patients in different stages of the disease.

\begin{tabular}{lcccc}
\hline Parameters & $\begin{array}{c}\text { Control } \\
(\mathbf{n}=20)\end{array}$ & $\begin{array}{c}\text { Stage 1\&2 } \\
(\mathbf{n}=20)\end{array}$ & $\begin{array}{c}\text { Stage 3 } \\
(\mathbf{n}=20)\end{array}$ & $\begin{array}{c}\text { Stage 4 } \\
(\mathbf{n}=20)\end{array}$ \\
\hline Total cholesterol $(\mathrm{mg} / \mathrm{dl})^{*}$ & $172.95 \pm 19.24$ & $203.70 \pm 30.91^{\dagger}$ & $216.75 \pm 29.34^{\ddagger \ddagger \ddagger}$ & $199.65 \pm 43.06^{\S}$ \\
HDL-c $(\mathrm{mg} / \mathrm{dl})^{*}$ & $42.55 \pm 7.85$ & $46.10 \pm 5.94$ & $48.15 \pm 11.43$ & $46.45 \pm 11.24$ \\
LDL-c $(\mathrm{mg} / \mathrm{dl})$ & $110.5(32)$ & $140(63.3)$ & $141.5(30)^{\ddagger \ddagger}$ & $119.5(47.5)$ \\
VLDL-c $(\mathrm{mg} / \mathrm{dl})$ & $25.50(14)$ & $25.50(25.5)$ & $21.0(9)$ & $24.0(13.5)$ \\
TG $(\mathrm{mg} / \mathrm{dl})$ & $128.0(78)$ & $126.5(127.3)$ & $110.0(52.25)$ & $122.0(65.75)$ \\
\hline
\end{tabular}

${ }^{*}$ Data expressed as mean \pm SD. All other data expressed as Median (Inter Quartile Range). ${ }^{\dagger}$ control vs. stage 182 ; ${ }^{\ddagger}$ control vs. stage $3 ;{ }^{\S}$ control vs stage $4 ;{ }^{\dagger,} p$-value $<0.05 ;{ }^{\ddagger \ddagger} p$-value $<0.01 ;{ }^{\ddagger \ddagger \ddagger} p$-value $<0.001$ 
A)

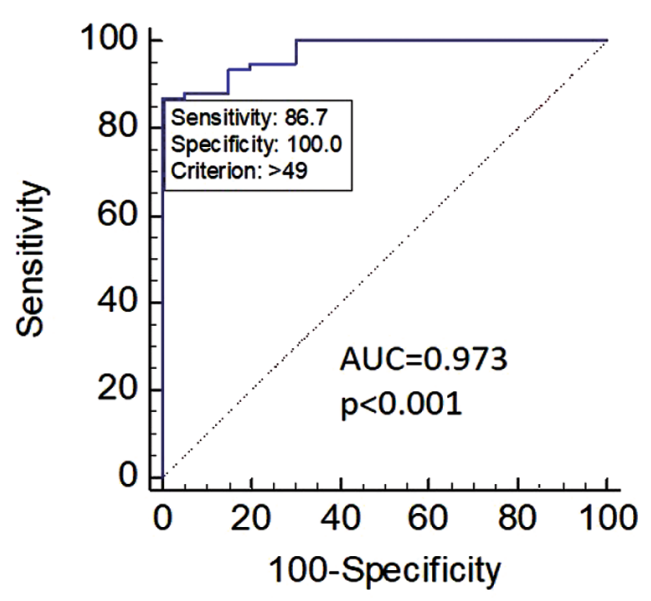

C)

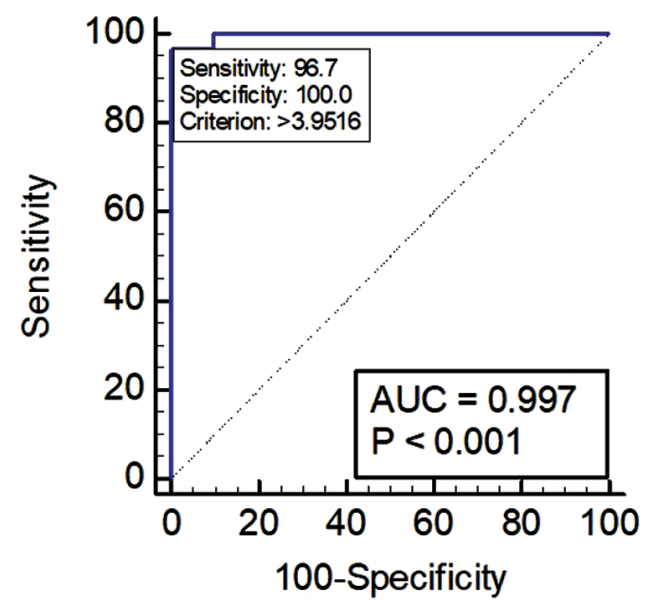

B)

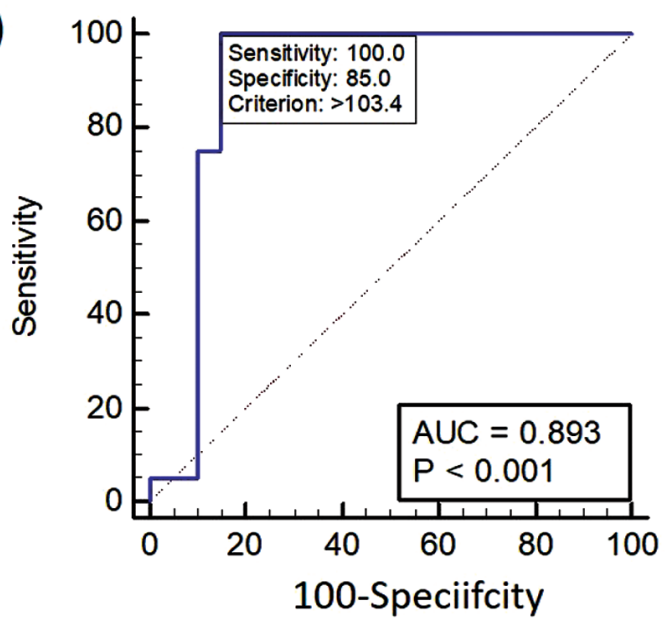

D)

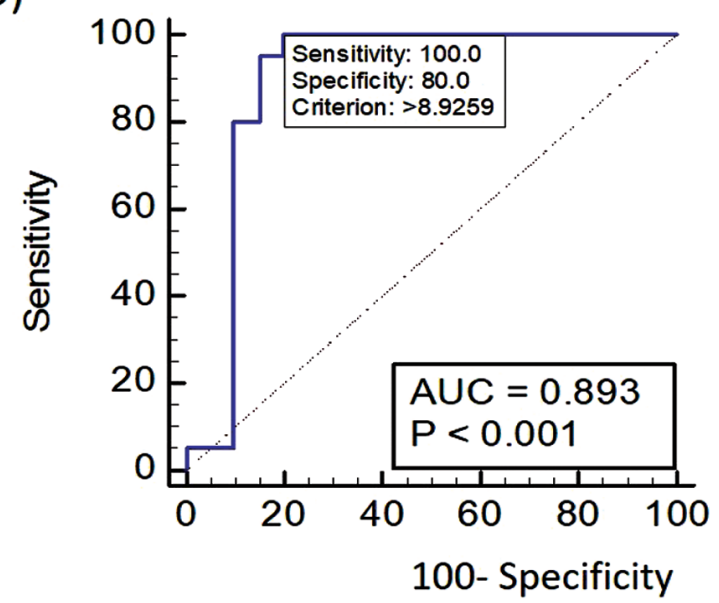

Figure 3 - Receiver operating characteristic curve analyses for the diagnosis and differentiation of the stages of breast cancer. A) Differentiation of healthy controls and breast cancer patients using Ferritin. B) Differentiation of stage 3 and stage 4 of breast cancer using Ferritin. C) Differentiation of healthy controls and breast cancer patients using Ferritin/Hb ratio. D) Differentiation of stage 3 and stage 4 of breast cancer using Ferritin/ $\mathrm{Hb}$ ratio. AUC: Area under curve

more beneficial for the patients, oncogene expression, histological grade of the tumor and status of hormone receptor are considered. ${ }^{2,3}$ However, anatomic staging is still being used when the expensive biomarker testing is not available. ${ }^{4}$ In this context, there is a need for the identification of less expensive markers. At present, the treatment strategies for breast cancer have been revolutionized due to the advances in the availability of individual's hormone receptor status and multiple gene panels. However, this becomes more fruitful when it is integrated with the prognostic staging of the disease.

We assessed the utility of serum ferritin and other inflammatory biomarkers, hsCRP and hepcidin in the diagnosis of different stages of breast cancer. Among the parameters of our study, we found ferritin as most useful in the diagnosis and staging of breast cancer. Jacobs et al reported higher ferritin concentrations among cancer patients. As per their study, the tumor recurrence is higher among those patients whose basal systemic ferritin levels are $>200 \mathrm{ug} / 1 .{ }^{6}$ This prognostic value of ferritin justifies its inclusion among the battery of biochemical and genetic parameters in the prognostic staging of breast cancer.

Our study reveals that ferritin and ferritin/Hb ratio are useful in the diagnosis of the different stages of breast cancer. However, these results need to be confirmed with a bigger sample size. The median value of ferritin among the breast cancer patients did not cross $200 \mathrm{ug} / \mathrm{l}$. Even the median value of serum ferritin among the healthy control of our study is only $29 \mathrm{ug} / \mathrm{l}$. This may 
be explained by the poor socioeconomic status of our patients. The ratio of ferritin/ $\mathrm{Hb}$ was chosen based on the fact that in breast cancer patients, when the ferritin levels rise, the $\mathrm{Hb}$ levels decrease.

Iron homeostasis is closely associated with the inflammatory processes. The serum ferritin levels increase through the upregulation of hepcidin mediated by IL-6. ${ }^{9-12}$ In our study, serum levels of hepcidin correlated positively with hsCRP, which is a marker of inflammation. In breast cancer, increased levels of ferritin have been observed, which could be due to excess iron stores, or the underlying inflammation, or both. ${ }^{13}$ The systemic ferritin serves as an index of inflammation or the body's iron stores. The elevated levels of ferritin in blood as well as in tumor tissues of breast cancer patients correlated with poor prognosis and the advanced histological grade. ${ }^{7}$ The breast cancer patients have higher plasma hepcidin levels which is not an observation in non-cancerous breast diseases. Sandhya et al reported the usefulness of ferritin in differentiating breast cancer patients with and without metastasis. ${ }^{14}$ The presence of malignancy can be identified by elevated ferritin levels and is useful in predicting lymph node involvement in breast cancer. ${ }^{15}$ The origin of serum ferritin in physiological and pathological conditions could be hepatocytes, macrophages, and cancer cells. ${ }^{11}$ Liver is the primary site of hepcidin synthesis. Infection and inflammation enhance the expression of hepcidin by the hepatocytes. ${ }^{16}$ In inflammation, the iron recycling from macrophages is inhibited by increased hepcidin levels. This increases serum ferritin levels, systemic iron deficiency and anemia. ${ }^{17,18}$ The breast cancer cells assimilate and retain surplus iron by enhancing the iron import and reducing the iron export. ${ }^{19}$

One of the crucial targets of cancer therapy is its glucose metabolism. The tumor cells, in general, manifests increased uptake of glucose in comparison to the neighboring normal cells. ${ }^{20,21}$ Iron is required as a micronutrient for glucose oxidation..$^{22}$ The iron metabolism is altered in tumors of the breast. ${ }^{3}$ The proteins and enzymes involved in iron homeostasis could serve as diagnostic and prognostic indicators as well as potential targets for therapy. In order to arrest disease progression, iron chelators could be administered. ${ }^{23}$ Further anti-inflammatory agents may arrest the progression of breast cancer.

The major outcome of this study is the identification of ferritin and inflammatory biomarkers as useful parameters in the diagnosis of different stages of breast cancer. One of the limitations of this study is that we did not collect data on the body mass index of the patients. The iron metabolism, ferritin, hepcidin and obesity are inter-related. The impaired metabolic functions observed in patients with type 2 diabetes are associated with iron overload and elevated levels of hepcidin. ${ }^{24}$ Obesity can induce inflammation which can disturb iron homeostasis by inhibiting its absorption, and by its retention in the reticuloendothelial system. The altered iron homeostasis can cause anemia and its related pathological complications. ${ }^{25,26}$ Activation of noncanonical Wnt signaling through the up-regulation of WNT5A may promote a proinflammatory state in the mammary adipose tissue contributing to the development of obesity-associated comorbidities. This has been shown in visceral adipose tissue. ${ }^{27,28}$ Aquaporin 7 operates as a glycerol channel in vivo. Adipocyte glycerol permeability has a key role in the regulation of fat accumulation. In view of this, inhibition of aquaporins have been suggested to be beneficial. ${ }^{29,30}$ Therefore, activation of noncanonical Wnt signaling and Aquaporin 7 are some of the important future areas of research in breast cancer.

In conclusion, biochemical derangements related to low-grade inflammation increased progressively in the disease progression of breast cancer. Assessment of ferritin, hepcidin, hsCRP as well as ferritin/ $\mathrm{Hb}$ ratio would be helpful in the differential diagnosis of different stages of breast cancer. Although the present study was carried out with a small cohort of patients, further studies with a larger sample size may be carried out to establish cut off values for the other indices of iron homeostasis like transferrin receptor-ferritin index (sTfR/log ferritin), and so forth.

Acknowledgment. The authors gratefully acknowledge Grammarly for the English language editing.

\section{References}

1. Petekkaya I, Unlu O, Roach EC, Gecmez G, Okoh AK, Babacan T, et al. Prognostic role of inflammatory biomarkers in metastatic breast cancer. J BUON 2017; 22: 614-622.

2. Gauldie J, Richards C, Harnish D, Lansdorp P, Baumann H. Interferon beta $2 / \mathrm{B}$-cell stimulatory factor type 2 shares identity with monocyte-derived hepatocyte-stimulating factor and regulates the major acute phase protein response in liver cells. Proc Natl Acad Sci US A 1987; 84: 7251-7255.

3. Colotta F, Allavena P, Sica A, Garlanda C, Mantovani A. Cancerrelated inflammation, the seventh hallmark of cancer: links to genetic instability. Carcinogenesis 2009; 30: 1073-1081.

4. Grivennikov SI, Greten FR, Karin M. Immunity, inflammation, and cancer. Cell 2010; 140: 883-899.

5. Kabat GC, Rohan TE. Does excess iron play a role in breast carcinogenesis? An unresolved hypothesis. Cancer Causes Control 2007; 18: 1047-1053.

6. Jacobs A, Jones B, Ricketts C, Bellbrook R. D and Wangt D. Y. Serum ferritin concentration in early breast cancer. Br J Cancer 1976; 34: 286. 
7. Alkhateeb AA, Han B, Connor JR. Ferritin stimulates breast cancer cells through an iron-independent mechanism and is localized within tumor-associated macrophages. Breast Cancer Res Treat 2013; 137: 733-744.

8. Asegaonkar S, Takalkar U, Kodlikeri P, Pagdhune A, Bonduliya V, Thorat A. Serum high sensitivity C-reactive protein in breast cancer patients. Int J Res Med Sci 2014; 2: 1408.

9. Kumari N, Dwarakanath BS, Das A, Bhatt AN. Role of interleukin-6 in cancer progression and therapeutic resistance. Tumor Biol 2016; 37: 11553-11572.

10. Esquivel-Velázquez M, Ostoa-Saloma P, Palacios-Arreola MI, Nava-Castro KE, Castro JI, Morales-Montor J. The Role of Cytokines in Breast Cancer Development and Progression. $J$ Interferon Cytokine Res 2015; 35: 1-16.

11. Ganz T. Hepcidin, a key regulator of iron metabolism and mediator of anemia of inflammation. Blood 2003; 102: 783-788.

12. Reuter S, Gupta SC, Chaturvedi MM, Aggarwal BB. Oxidative stress, inflammation, and cancer: How are they linked? Free Radic Biol Med 2010; 49: 1603-1616.

13. Macciò A, Madeddu C, Gramignano G, Mulas C, Tanca $\mathrm{L}$, Cherchi MC, et al. The role of inflammation, iron, and nutritional status in cancer-related anemia: results of a large, prospective, observational study. Haematologica 2015; 100: 124-132.

14. Amrita V,P Sarkar, K sodavadia.Serum Iron And Ferritin As Diagnostic Marker of Breast Cancer. Natl J Integr Res Med 2018; 9: 1-6.

15. Ulbrich EJ, Lebrecht A, Schneider I, Ludwig E, Koelbl H, Hefler LA. Serum parameters of iron metabolism in patients with breast cancer. Anticancer Res 2003; 23: 5107-5109.

16. Deschemin JC, Vaulont S. Role of hepcidin in the setting of hypoferremia during acute inflammation. PLos One 2013; 8: e61050.

17. Sukhbaatar N, Weichhart T. Iron Regulation: Macrophages in Control. Pharmaceuticals (Basel) 2018; 11: 137.

18. Gammella E, Buratti P, Cairo G, Recalcati S. Macrophages: central regulators of ironbalance. Metallomics 2014; 6: 1336-1345.

19. Orlandi R, De Bortoli M, Ciniselli CM, Vaghi E, Caccia D, Garrisi V, et al. Hepcidin and ferritin blood level as noninvasive tools for predicting breast cancer. Ann Oncol 2014; 25:352-357.
20. Bose S, Le A. Glucose metabolism in cancer. Adv Exp Med Biol 2018; 1063: 3-12.

21. Annibaldi A, Widmann C. Glucose metabolism in cancer cells. Curr Opin Clin Nutr Metab Care 2010; 13: 466-470.

22. Carlos AR, Weis S, Soares MP. Cross-talk between iron and glucose metabolism in the establishment of disease tolerance. Front Immunol 2018; 9: 2498.

23. Yu Y, Gutierrez E, Kovacevic Z, Saletta F, Obeidy P, Suryo Rahmanto Y, et al. Iron chelators for the treatment of cancer. Curr Med Chem 2012; 19: 2689-26702.

24. Ndevahoma F, Mukesi M, Dludla PV, Nkambule BB, Nepolo EP, Nyambuya TM. Body weight and its influence on hepcidin levels in patients with type 2 diabetes: A systematic review and meta-analysis of clinical studies. Heliyon 2021; 7: e06429.

25. Becker C, Orozco M, Solomons NW, Schümann K. Iron metabolism in obesity: how interaction between homoeostatic mechanisms can interfere with their original purpose. Part I: underlying homoeostatic mechanisms of energy storage and iron metabolisms and their interaction. J Trace Elem Med Biol 2015; 30: 195-201.

26. Nikonorov AA, Skalnaya MG, Tinkov AA, Skalny AV. Mutual interaction between iron homeostasis and obesity pathogenesis. J Trace Elem Med Biol 2015; 30: 207-214.

27. Catalán V, Gómez-Ambrosi J, Rodríguez A, et al. Activation of noncanonical Wnt signaling through WNT5A in visceral adipose tissue of obese subjects is related to inflammation. $J$ Clin Endocrinol Metab 2014; 99: E1407-E1417.

28. Ma W, Jia L, Xiong Q, Feng Y, Du H. The role of iron homeostasis in adipocyte metabolism. Food Funct 2021; 12: 4246-4253.

29. Frühbeck G. Obesity: aquaporin enters the picture. Nature 2005; 438: 436-437.

30. Alkhalifa H, Mohammed F, Taurin S, Greish K, Taha S, Fredericks $S$. Inhibition of aquaporins as a potential adjunct to breast cancer cryotherapy. Oncol Lett 2021; 21: 458. 